\title{
Lagrangian velocity statistics of directed launch strategies in a Gulf of Mexico model
}

\author{
M. Toner ${ }^{1}$ and A. C. Poje ${ }^{2}$ \\ ${ }^{1}$ College of Marine Studies, University of Delaware, Newark, Delaware, USA \\ ${ }^{2}$ Department of Mathematics, College of Staten Island, Staten Island, New York, USA \\ Received: 17 March 2003 - Revised: 6 June 2003 - Accepted: 10 June 2003 - Published: 25 February 2004 \\ Part of Special Issue "Dedicated to Prof. A. D. Kirwan Jr. on the occasion of his 70th birthday"
}

\begin{abstract}
The spatial dependence of Lagrangian displacement and velocity statistics is studied in the context of a data assimilating numerical model of the Gulf Mexico. In the active eddy region of the Western Gulf, a combination of Eulerian and Lagrangian measures are used to locate strongly hyperbolic regions of the flow. The statistics of the velocity field sampled by sets of drifters launched specifically in these hyperbolic regions are compared to those produced by randomly chosen launch sites. The results show that particle trajectories initialized in hyperbolic regions preferentially sample a broader range of Eulerian velocities than do members of ensembles of randomly launched drifters. The velocity density functions produced by the directed launches compare well with Eulerian velocity pdfs. Implications for the development of launch strategies to improve Eulerian velocity field reconstruction from drifter data are discussed.
\end{abstract}

\section{Introduction}

The goal of this work is to examine how Lagrangian velocity statistics depend on initial launch position in the context of a realistic ocean model. Given the importance of Lagrangianbased oceanic observations, questions concerning the distributions of velocities sampled along drifter and float trajectories have direct bearing on both the design of optimal Lagrangian observing systems and the fidelity of Eulerian fields reconstructed from such data using objective mapping techniques (Eremeev et al., 1992; Toner et al., 2001b; Poje et al., 2002) or generated through data assimilative models (Kamachi and O'Brien, 1995; Ishikawa et al., 1996; Molcard et al., 2003).

The long time distributions and spectra of Lagrangian velocity statistics derived from direct oceanic observations and large-scale numerical models have been the subject of a number of recent studies (Rupolo et al., 1996; Bracco et al., 2000;

Correspondence to: $\mathrm{M}$. Toner

(toner@udel.edu)
Garraffo et al., 2001). In agreement with observations and analysis of comparatively simple, two-dimensional turbulence models, the presence of persistent energetic structures in oceanic flow fields leads to regimes of anomalous particle dispersion and highly non-Gaussian velocity distributions in the Lagrangian frame (see, for example Provenzale, 1999, and references therein).

Structure-dominated flow fields are typically characterized by a distinct separation between slow Eulerian and fast Lagrangian velocity auto-correlation time-scales (Rupolo et al., 1996; Hua et al., 1998; Poje et al., 1999) leading to so-called Lagrangian chaos in the particle dynamics. Given the disparity of time-scales in the Eulerian and Lagrangian frames, the material boundaries of Lagrangian coherent structures can be identified in terms of stable (in-flowing) and unstable (outflowing) manifolds associated with finite time hyperbolic trajectories (Haller and Poje, 1998). These boundary curves provide a precise partition of the flow into regions of different dynamic behaviour.

In this work we investigate how well drifters sample the known Eulerian velocity distribution based on their launch location for relatively short, weekly time scales of interest for data assimilation and ocean now-casting purposes. The goal is to quantify this sampling based on the proximity of the launch positions to material boundaries of Lagrangian coherent structures in the flow. To this end, we study two dimensional velocity fields produced by a realistic, data assimilating model of the Gulf of Mexico. In particular, we focus on the eddy structure in the western region of the Gulf.

\section{Circulation in the Gulf}

The Gulf of Mexico is an ideal numerical laboratory because of its relatively small size and semi-enclosed nature. At any time, between 23-28 Sverdrups of sub-tropical water from the Caribbean enter the Gulf through the Yucatan Strait (see Fig. 1), intrude into the Gulf as the Loop Current, and then exit through the Straits of Florida to become 

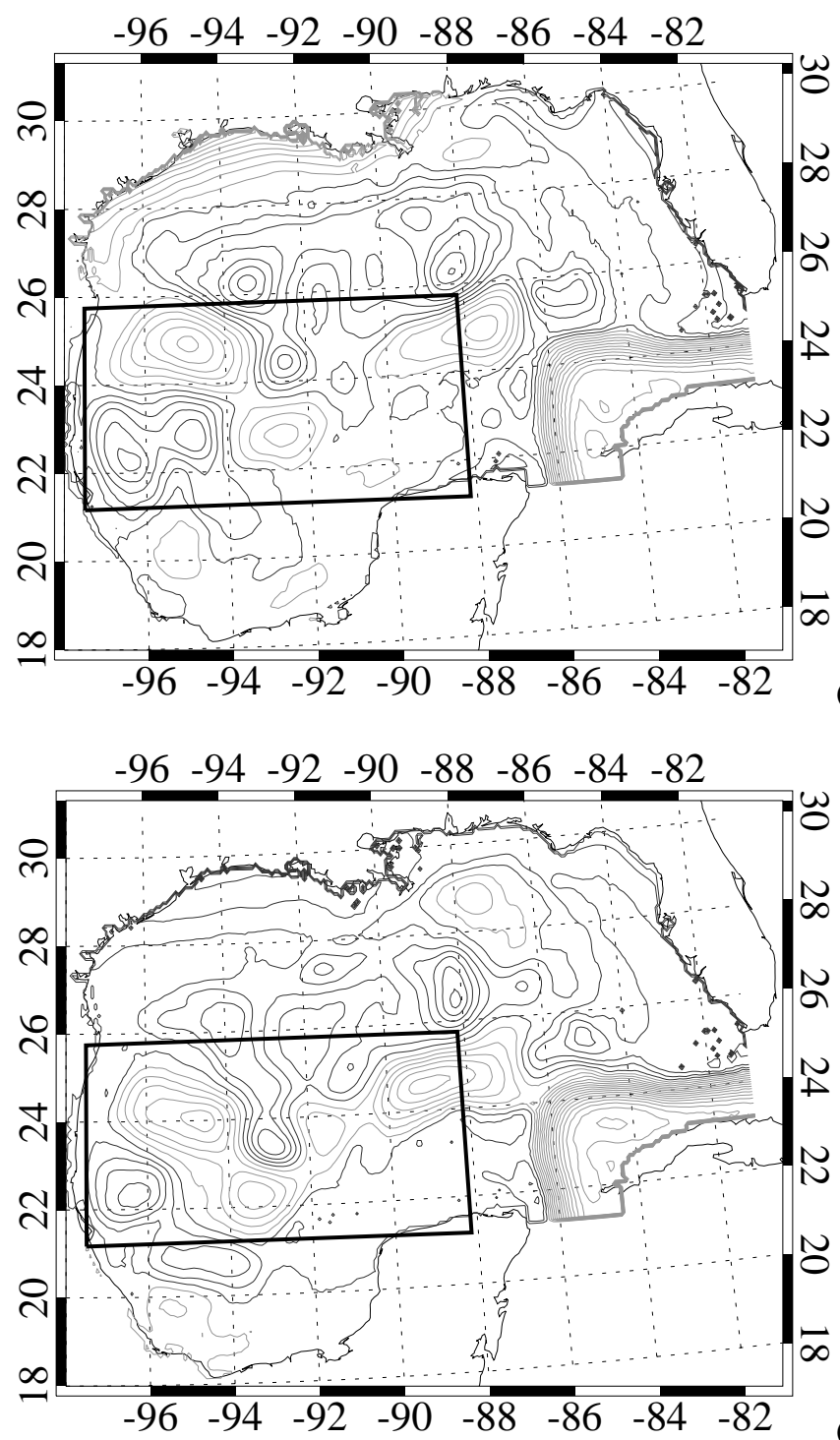

Fig. 1. Contours of the model SSH field at the beginning and end of the deployment period. Grey and black contours indicate positive and negative $\mathrm{SSH}$ anomalies (taken from rest) respectively. The region of interest for the study is shown in the western Gulf. (a) 18 October, (b) 17 November.

the Florida Current and eventually the Gulf Stream. Episodically, the Loop Current sheds highly energetic anticyclonic rings which migrate westward. The entire Gulf is thus, driven by the variability associated with the Loop Current and its rings. The currents in the Loop Current and the Loop Current Eddies can exceed 4 knots $(2 \mathrm{~m} / \mathrm{s})$. Associated with the Loop Current rings are smaller, but intense, cyclones with swirl velocities as large as $0.5 \mathrm{~m} / \mathrm{s}$. Thermal signatures of both the rings and cyclones are apparent at $900 \mathrm{~m}$ (Lewis and Kirwan, 1985).

To study the Lagrangian dynamics in the Gulf region, we use velocity fields produced by a state-of-the-art primitive equation model, CUPOM, developed at the University of
Colourado (Kantha et al., 1999). The model is a variant of the Princeton Ocean Model (Blumberg and Mellor, 1987) which incorporates high order mixed layer and turbulence closure schemes and assimilates remotely-sensed MCSST and altimetric SSH anomaly data (Lopez and Kantha, 2000). The model evolves 24 sigma levels with horizontal resolution of 1/12 degree. Along-track TOPEX/Poseidon and ERS-2 altimeter data are assimilated as pseudo expendable bathythermographs using an optimal interpolation based data assimilation scheme. Near-real-time sea surface temperature is inferred from multi-channel infrared imagery. The surface is forced by the Navy Operational Global Atmospheric Prediction System (NOGAPS) winds. Modification (Kantha and Clayson, 1994) of the original POM mixed layer parameterization (Galperin et al., 1988) improves depiction of nearsurface currents, SST, and upper layer heat content. Inflow through the Yucatan Channel is prescribed based on climatological values, while outflow through the Florida Straits evolves with model physics.

The fidelity of the model has been tested in a number of hind-cast scenarios with remarkably good results. Toner et al. (2001a) directly compared near surface drifter trajectories within a large Loop Current ring with numerical trajectories from the CUPOM. Near surface drifters were also used by Kuznetsov et al. (2002) in conjunction with Lagrangian boundaries of coherent features in the model to verify complicated interactions of the Loop Current and adjacent cyclones and anti-cyclones. Accurate representations of the relative positions of cyclones and anti-cyclones was also demonstrated by Toner et al. (2003) who also used Lagrangian boundaries obtained from model currents to identify inter-eddy channels observed by remotely sensed ocean colour data (SeaWiFS) as they occurred.

Since we are concerned with inhomogeneities introduced (b) by the presence of interacting cyclones and anti-cyclones, we concentrate our analysis on the eddy field in the Western Gulf away from the direct influence of the dominant Loop Current (see Fig. 1). The flow in this deep water region is dominated by the evolution of a distinct number long-lived, large-scale $(\mathcal{O}(100 \mathrm{~km}))$ eddies that evolve on relatively long (weeklymonthly) time scales.

Particle trajectories in this study are found by integrating

$$
\begin{aligned}
& \frac{d x}{d t}=u(x, y, t) \\
& \frac{d y}{d t}=v(x, y, t)
\end{aligned}
$$

where $(u, v)$ are the zonal and meridional velocity components given by the model at $50 \mathrm{~m}$. This depth is representative of the near surface flow and is a typical drifter drogue depth (Toner et al., 2001a; Kuznetsov et al., 2002). Trajectory integration is performed with the LSODA routine from the public domain library ODEPACK using bicubic spatial and linear temporal interpolation of the gridded daily model velocities. 


\subsection{Time scales}

That the dynamics of Lagrangian tracers is dominated by energetic coherent features in the Eulerian eddy field can be seen by comparing the velocity auto-correlation time-scales for the Lagrangian and Eulerian fields. To test disparity between the Eulerian and Lagrangian time scales, we calculate the Eulerian velocity correlations directly from the model data and the Lagrangian velocity correlations from a subset of 15614 particle trajectories initialized at model grid points on $t_{0}=3$ October 1998 and integrated for 45 days.

The velocity auto-correlation functions defined by

$$
R_{\text {Lag }}\left(t ; t_{0}\right)=\left\langle\mathbf{v}\left(t_{0}, \mathbf{x}_{0}\right) \cdot \mathbf{v}\left(t_{0}+t, \mathbf{x}_{0}\right)\right\rangle /\left\langle\left|\mathbf{v}\left(t_{0}, \mathbf{x}_{0}\right)\right|^{2}\right\rangle
$$

and

$$
R_{E u l}\left(t ; t_{0}\right)=\overline{\mathbf{u}\left(\mathbf{x}, t_{0}\right) \cdot \mathbf{u}\left(\mathbf{x}, t_{0}+t\right)} / \overline{\left|\mathbf{u}\left(\mathbf{x}, t_{0}\right)\right|^{2}}
$$

are shown in Fig. 2a. Here the Lagrangian velocity along a trajectory, as a function of the initial position $\mathbf{x}_{0}$, is denoted by $\mathbf{v}\left(t, \mathbf{x}_{0}\right)$, while the Eulerian velocity field is denoted by $\mathbf{u}(\mathbf{x}, t)$. The Lagrangian average, $\langle(\cdots)\rangle$, is taken over all trajectories (14617) that do not leave the Gulf during the integration. The Eulerian average, $\overline{(\cdots)}$, is taken over the (fixed) spatial domain corresponding to the initial conditions $\left(\mathbf{x}_{0}\right)$ used in the Lagrangian average. Figure $2 \mathrm{~b}$ shows the resulting velocity autocorrelation times given by

$T_{\text {Lag }}\left(t ; t_{0}\right)=\int_{t_{0}}^{t_{o}+t_{\infty}} R_{\text {Lag }}\left(t ; t_{0}\right) d t$

and

$T_{E u l}\left(t ; t_{0}\right)=\int_{t_{0}}^{t_{o}+t_{\infty}} R_{E u l}\left(t ; t_{0}\right) d t$.

where $t_{\infty}=45$ days.

Clearly, the Lagrangian time scale, $T_{\text {Lag }} \approx 2.2$ days is considerably shorter than the corresponding Eulerian measure, which did not converge during the time interval of the computation. The difference between the two time scales is most simply explained by the presence of robust coherent vortex-structures that evolve relatively slowly in the Eulerian frame while rapidly stirring fluid particles. These conditions are fairly typical of meso-scale ocean flows containing isolated and energetic Eulerian structures and point to the existence of identifiable hyperbolic trajectories in the Lagrangian frame with attending Lagrangian coherent structure boundaries. This disparity of time-scales is also indicative of Lagrangian motion where small-scale advective exchange within and between eddies is confined to long thin filaments as defined by adiabatic theory (see, for example, Lebovitz and Neishtadt, 1994).

\subsection{Eulerian and Lagrangian partitions}

The coherent vortex features seen in Fig. 1 act as traps for particle motion and thus, strongly affect the distribution of

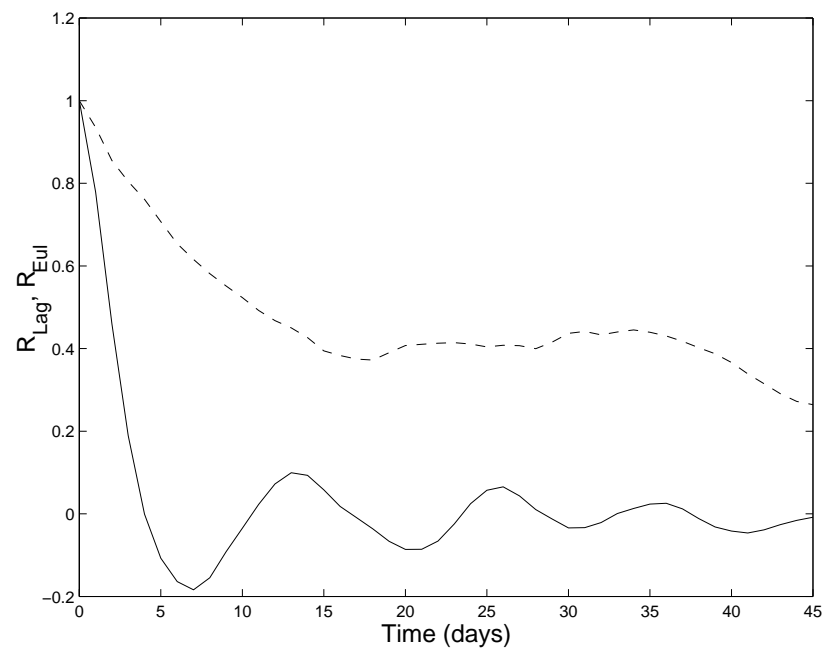

(a) Autocorrelation

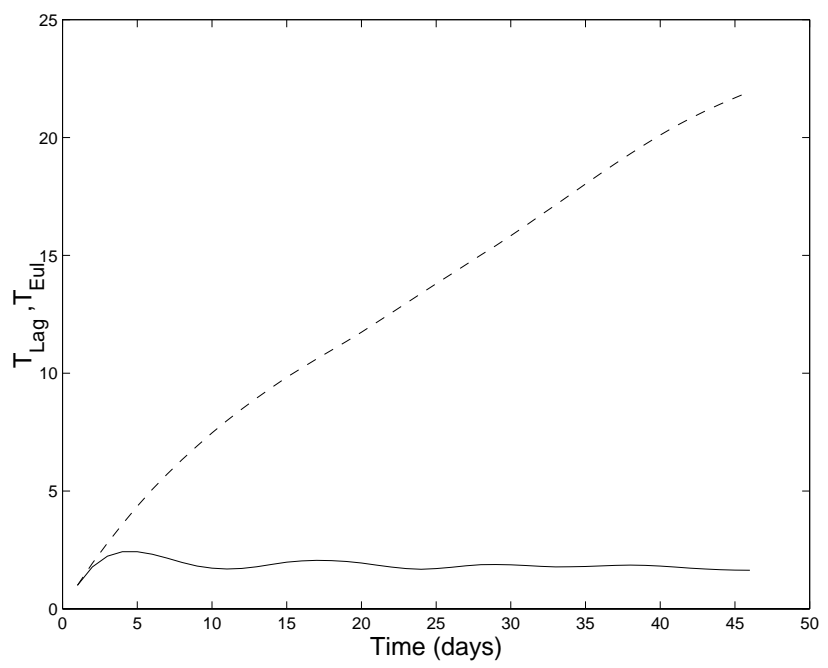

(b) Timescales

Fig. 2. Eulerian and Lagrangian velocity autocorrelation functions and time-scales for the model, computed on the portion of the model grid for which trajectories do not escape from the Gulf during the 45 day calculation period. This excludes the Loop Current and some adjacent regions, but contains the region of interest.

Lagrangian velocities sampled by different trajectories. Understanding the extent of particle trapping by eddy structures and the amount of particle exchange among features with different velocity signatures is crucial to understanding overall Lagrangian velocity sampling.

Partition of a complex flow field by identifying coherent features can be accomplished in both the Eulerian and Lagrangian frames. A relatively straightforward Eulerian separation based on the instantaneous structure of the velocity gradient tensor was proposed by Okubo (1970) and Weiss 


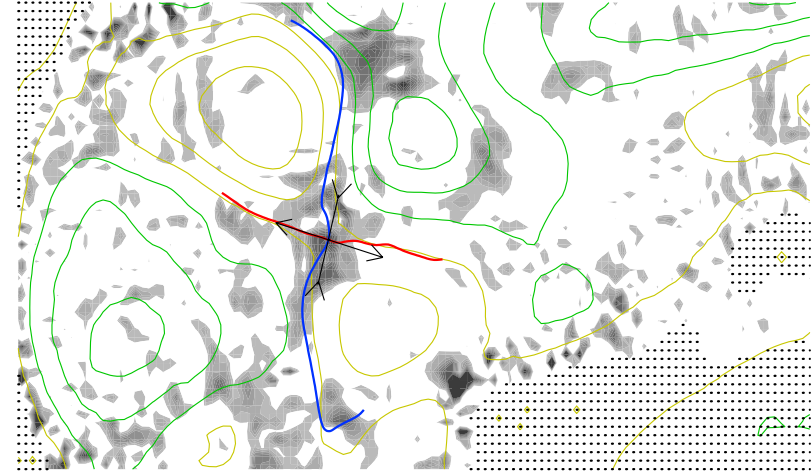

(a) Oct. 29

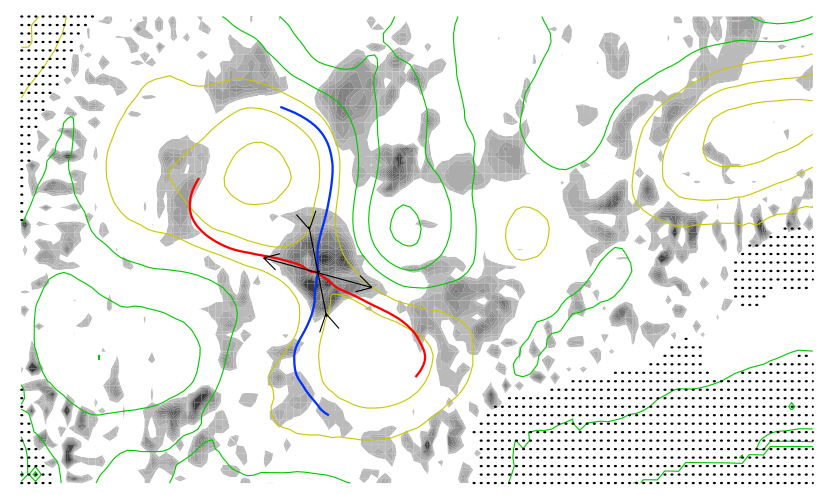

(b) Nov. 11

Fig. 3. Eulerian, Okubo-Weiss, partition of the flow for two days during the study. Greyscale regions indicate positive $Q$ with the darkest shade representing values that exceed $1.4 \times 10^{-10} \mathrm{~s}^{-2}\left(1 \mathrm{day}^{-2}\right)$. Superimposed are the model SSH contours at $7.5 \mathrm{~cm}$ intervals (yellow and green indicate positive and negative SSH anomalies respectively) and the in-flowing (blue) and out-flowing (red) material curves associated with the hyperbolic trajectory of interest. These material curves have been truncated to show the evolution of a small segment that lied across the hyperbolic trajectory four days before (out-flowing) and four days after (in-flowing) the indicated dates. Eigenvectors are indicated by the arrows emanating from the hyperbolic trajectory.

(1991) (see also Haller, 2001; Lapeyre et al., 1999). The Okubo-Weiss criterion compares the square of the local strain rate, $S$, to the vorticity, $\omega$, via the quantity

$Q(x, y, t)=S^{2}-\omega^{2}=-\operatorname{det}(\nabla \mathbf{u})$.

While only exact for particle trajectories in the limit of frozen, time independent, velocity fields, $Q$ measures the rate of local divergence of nearby trajectories in hyperbolic regions $(Q>0)$ and differentiates such stretching regions dominated by the local strain from elliptic regions $(Q<0)$ dominated by vorticity.
The spatial distribution of $Q$ computed using second order finite-differences of the model velocity field is shown in grey-scale for two different times in Fig. 3. Darker colours indicate hyperbolic regimes outside strongly elliptic regions marked in white at the eddy cores. The largest values of $Q\left(\sim 1\right.$ day $\left.^{-2}\right)$ are concentrated in the saddle regions of the dynamic height field between persistent like-signed vortex pairs.

The disparity of time-scales and the presence of strong, slowly evolving hyperbolicity in the Eulerian frame indicate the existence of well defined finite-time hyperbolic trajectories in the full, time-dependent, Lagrangian flow. As shown in Haller and Poje (1998), the Lagrangian hyperbolic trajectories are, at any time, located in the neighborhood of persistent saddle points in the corresponding frozen time Eulerian streamfunction. Once such trajectories are located, Lagrangian coherent structure boundaries (given by finitetime analogs of the stable and unstable manifolds of the hyperbolic trajectory) can be found by straddling techniques (Miller et al., 1997). Truncated segments of these bounding material curves are shown for one hyperbolic trajectory in Fig. 3 along with the eigenvectors of the deformation rate evaluated at the frozen time Eulerian saddle point. The manifold segments in each image shows the evolution of small $(\sim 30 \mathrm{~km})$ material curves that straddle the hyperbolic trajectory four days before (out-flowing) or after (in-flowing) the indicated dates. Stretching and compression rates based on the length of these curves show sustained values of about 0.6 day $^{-1}$. This value is somewhat smaller than the eigenvalue magnitudes at the hyperbolic trajectory $\left(\sim 1\right.$ day $\left.^{-1}\right)$ but more representative of oceanic drifters.

Unlike the Okubo-Weiss partition, finite-time stable and unstable manifolds of hyperbolic particle trajectories are invariant under the Lagrangian flow and provide an exact partition of fluid particle dynamics. Starting with the work of Aref (1984); Rom-Kedar et al. (1990) and MacKay et al. (1984) in strictly period velocity fields, the determination of manifold geometry has shed light on particle fluxes and stirring in increasingly complex oceanic flows (Ridderinkkhof and Loder, 1994; Poje and Haller, 1999; Coulliette and Wiggins, 2000; Miller et al., 2002; Kuznetsov et al., 2002). In the next two sections, connections between the geometry of Lagrangian coherent structure boundaries and distributions of particle dispersion and velocity statistics are examined.

\section{Dispersion statistics}

Elhmaidi et al. (1993) used the Okubo-Weiss partition to condition particle dispersion statistics in barotropic turbulent flow. Single particle dispersion statistics at intermediate times are strongly influenced by initial conditions with those launched from elliptic regions obeying different scaling laws than those launched in strain dominated regions. The connection between Lagrangian coherent features and two-particle dispersion statistics is even stronger. Given the near exponential stretching of line elements straddling the 


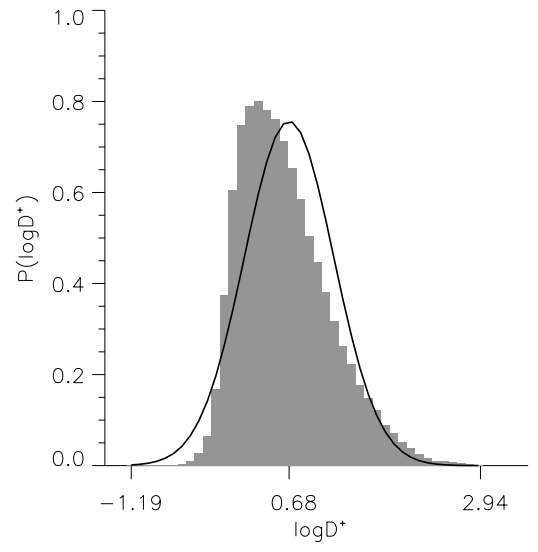

(a) Positive Dispersion

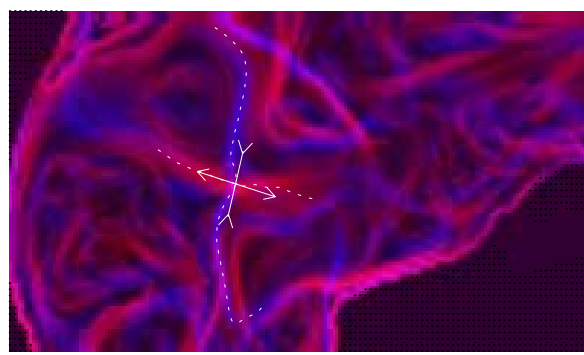

(c) Oct. 29

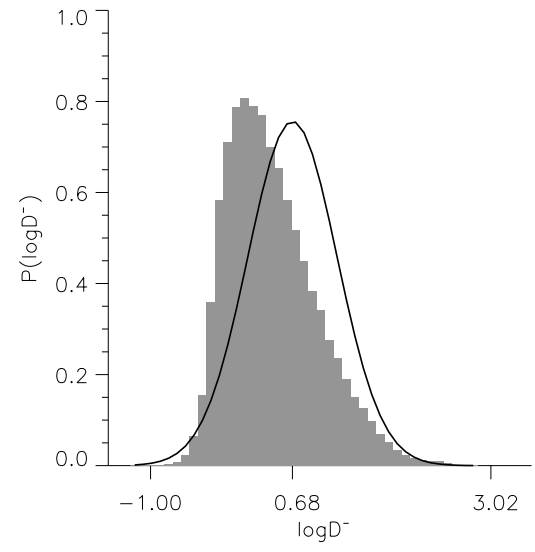

(b) Negative Dispersion

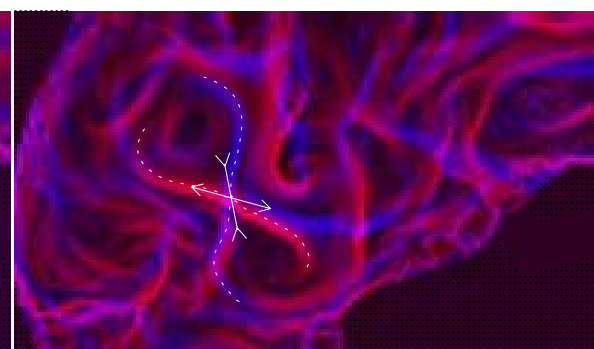

(d) Nov. 11
Fig. 4. Density and spatial distributions of normalized two particle dispersion computed for $\tau=$ five days from trajectories initialized on the model grid. The solid line shows log-normal distribution with the same mean and variance as the data. The spatial distributions shown in (c) and (d) show positive dispersion in blue and negative dispersion in red, with the maximum and minimum values approximately the same as indicated by the histograms (see Table 1). Superimposed on these images in dotted lines are the same material curve segments described in the caption of Fig. 3. stable manifold in the vicinity of a hyperbolic trajectory, Bowman (1999) proposed plotting the finite-strain associated with nearest neighbor particle separations as means of locating hyperbolic structures in complex, translating atmospheric flows. Using this approach, Jones and Winkler (2001) determined the location of hyperbolic trajectories in observed wind data and found that the spatial structure of relative dispersion was a good approximation for finite-time/size Lyapunov exponents which also have been used to visualize hyperbolic trajectories (Pierrehumbert, 1991; Boffetta et al., 2001).

To determine the finite strain, trajectories are initialized on a regular grid of initial conditions where $\mathbf{x}_{i, j}\left(t_{0}\right)$ indicates the initial position of the $i, j$ particle and $\mathbf{x}_{i, j}\left(t ; t_{0}\right)$ the subsequent position at time $t+t_{0}$. The normalized relative dispersion of nearest neighbor particles surrounding the point $\mathbf{x}_{i+1 / 2, j+1 / 2}\left(t_{0}\right)$ is calculated via the quantity

$$
\begin{aligned}
& 2 \Delta_{i, j}^{2} D_{i+1 / 2, j+1 / 2}^{ \pm}\left( \pm \tau ; t_{0}\right) \\
& \quad=\left\|\left(\mathbf{x}_{i+1, j+1}\left( \pm \tau ; t_{0}\right)-\mathbf{x}_{i, j}\left( \pm \tau ; t_{0}\right)\right)\right\|^{2} \\
& \quad+\left\|\left(\mathbf{x}_{i+1, j}\left( \pm \tau ; t_{0}\right)-\mathbf{x}_{i, j+1}\left( \pm \tau ; t_{0}\right)\right)\right\|^{2}
\end{aligned}
$$

where $\Delta_{i, j}$ is the initial separation distance between particle pairs. For integration times $\tau$ on the order of several Lagrangian correlation times, the field $D^{ \pm}\left(\tau ; t_{0}\right)$ plotted over the initial conditions provides information about the Lagrangian structure of the flow. For forward in time computations, peaks in the relative dispersion should identify segments of in-flowing manifolds associated with any nearby hyperbolic trajectory in the flow. Adjacent trajectories straddling the in-flowing manifold and approaching the hyperbolic region will experience near exponential separation rates along opposite legs of the associated out-flowing manifold. Conversely, computing the relative dispersion of trajectories advected backward in time highlights segments of the out-flowing manifold.

Figure 4 shows histograms of $D^{ \pm}(x, y ; \tau=5$ days) for a grid of approximately 14000 initial conditions. As indicated by the logarithmic scale in the figure, the relative dispersion distribution is skewed from log-normal with extremely long tails in the large separation region. Select particle pairs experience stretching orders of magnitude higher than the median value. The Lagrangian mean value of the relative dispersion is thus, strongly influenced by relatively few, superdispersive, trajectory pairs. As shown by the spatial distribution of the logarithm of $D$ in Figs. $4 \mathrm{c}$ and d, these pairs are precisely those that straddle the Lagrangian structures in the flow. The similarity between the relative dispersion distributions in forward and backward time is a result of the relatively long Eulerian time-scales (the advecting velocity field is qualitatively similar on the $\sim 30$ day time-scale) and the near 2-D incompressibility of the model velocity data 


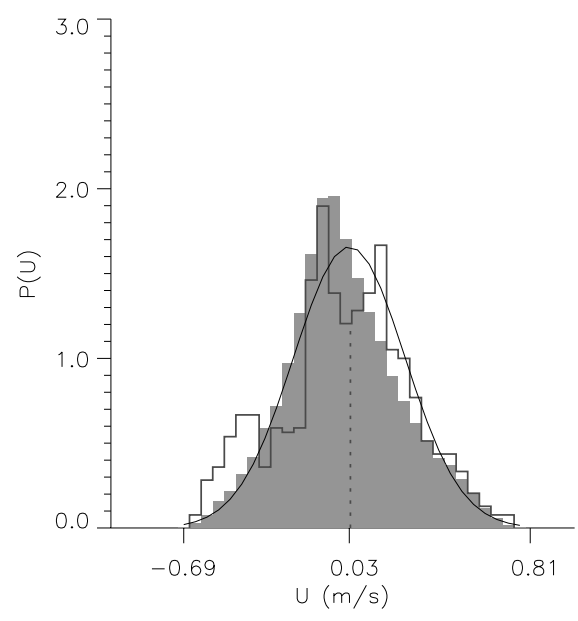

(a) Directed launch

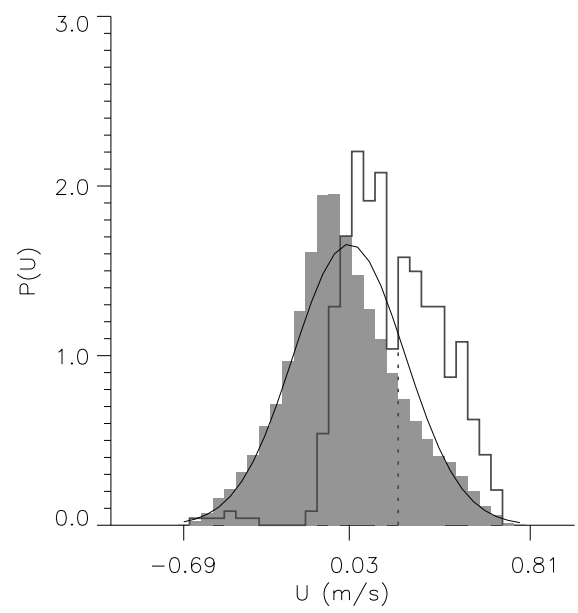

(c) Worst random launch

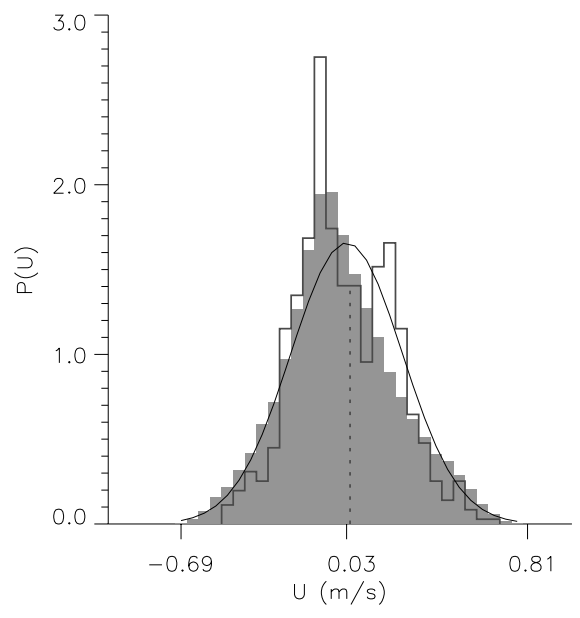

(b) Best random launch

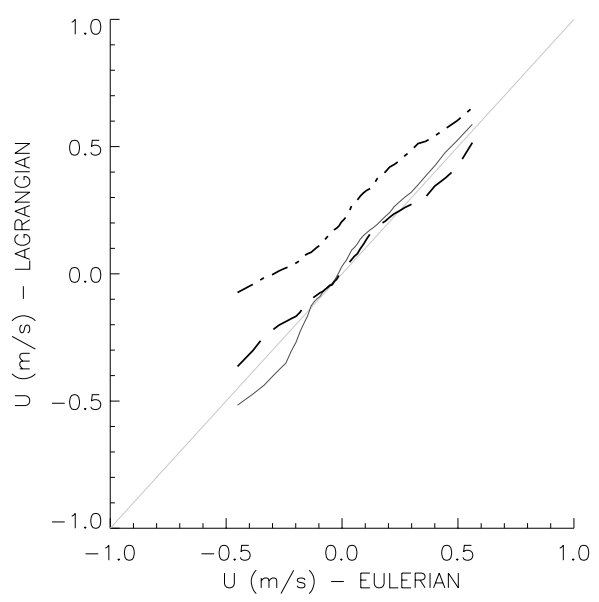

(d) Quantile-quantile plot

Fig. 5. Probability density functions (a)-(c) of zonal velocity sampling and quantile-quantile plots (d) for the directed, best, and worst random deployments. The error metric used is based on the quantile-quantile plot of the Lagrangian verses Eulerian histograms (before mapping into PDFs). The velocities indicated on the ordinate in (a)-(c) are the minimum, mean, and maximum values from the Eulerian sampling; the mean of the Lagrangian velocity sampling is indicated by the vertical dotted line. See Table 1 for more details on the statistics. In (d), the solid, dashed, and dash-doted lines represent the directed, best random, and worst random deployments respectively; the grey line represents the plot of a "zero" error sampling.

(positive and negative eigenvalues of the local rate of strain are nearly equal in magnitude).

A launch strategy based on locating Lagrangian hyperbolic trajectories is examined in the next section (see also Poje et al., 2002).

\section{Velocity statistics}

On weekly time scales, sampling the Eulerian velocity distribution with drifters will be strongly effected by coherent features in the flow. Knowledge of the location of these features can drastically improve how well the velocity distribution sampled by the drifters mimic the true, Eulerian distribution. Hyperbolic trajectories which may be easily identified in the Gulf of Mexico are associated with very low velocity regions, and clearly this trajectory alone will not sample the Eulerian PDF well. However, nearly every nearby trajectory (with the exception of those on the stable manifold) will exit this low velocity region rapidly (exponentially fast in the linearized saddle dynamics). Practically speaking, it will be impossible for a particle not to exit along a branch of the out-flowing manifold due to either numerical imprecision associated with model trajectories or small-scale turbulence and/or wind effects in the ocean. In the context 


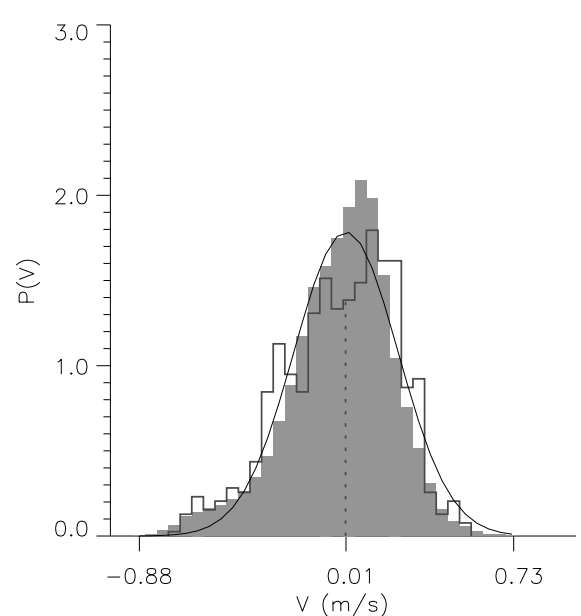

(a) Directed launch

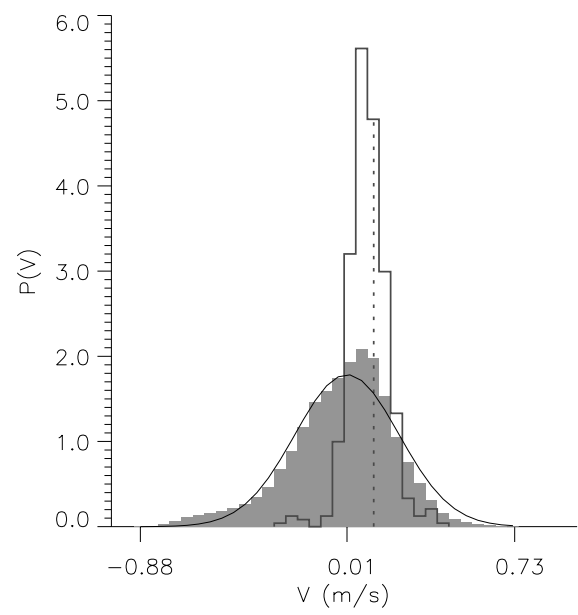

(c) Worst random launch

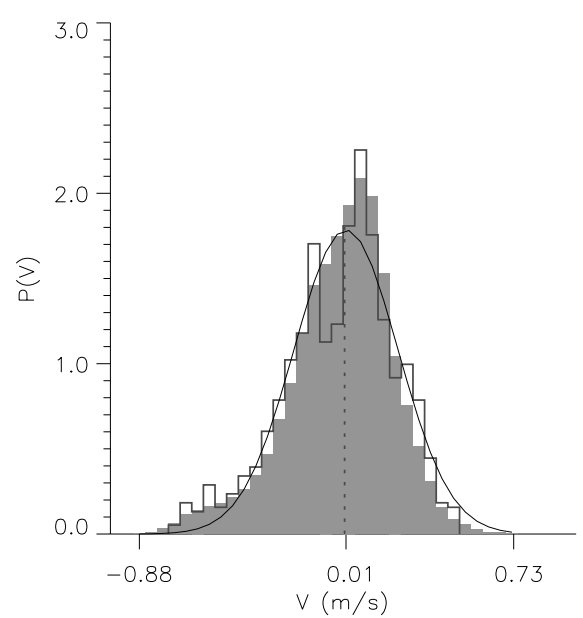

(b) Best random launch

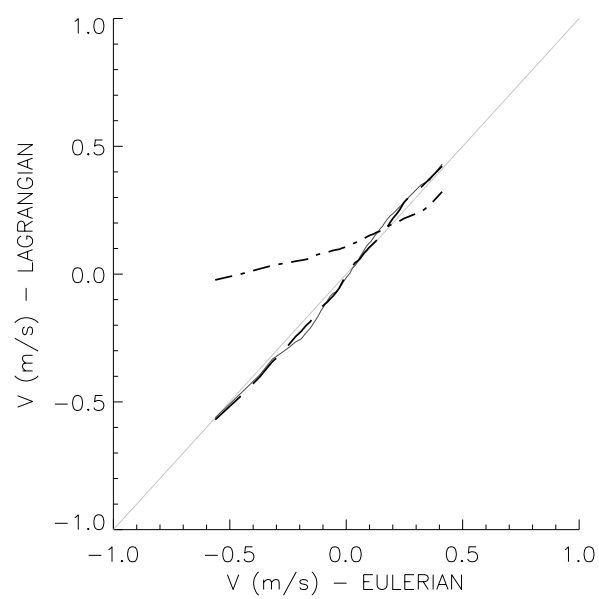

(d) Quantile-quantile plot

Fig. 6. Same as in Fig. 5 except for meridional velocity.

of mesoscale ocean eddies, out-flowing manifolds will form the Lagrangian boundaries of the coherent features and these boundaries tend to pass through very high velocity regions of the flow. Therefore, drifters launched in the vicinity of hyperbolic trajectories should experience both velocity extremes inherent in the flow. How well the resulting PDF generated from the Lagrangian velocities match the Eulerian PDF is addressed in the next sections.

\subsection{Launch strategy}

The region and time span of the velocity sampling in the Gulf of Mexico are shown in Fig. 1. A directed launch strategy based on knowledge of the hyperbolic trajectory shown in Figs. 3 and 4 is used to sample this region. To locate and track this trajectory, a small (approximately $20 \mathrm{~km}$ ) material line segment was initialized on 18 October along the out-flowing direction (as determined by the velocity gradient and geometric orientation of $D^{-}$on that day) and integrated forward in time until 17 November. In a similar fashion, a material line segment was initialized on 17 November along the in-flowing direction and integrated backward in time to 18 October. The appropriate intersection of these two curves was tracked throughout this time period and thus, located the hyperbolic trajectory. Segments of these manifolds are shown in Figs. 3 and 4. Beginning on 19 October and ending on 17 November, drifters were launched daily on either side of this intersecting point, approximately $30 \mathrm{~km}$ apart, along the out-flowing direction, as determined by the location of the out-flowing manifold near the hyperbolic trajectory at that time. As seen in Fig. 1, translation of the launch location was minimal (approximately $50 \mathrm{~km}$ ).

For comparison, 20 random locations were chosen well off the continental shelf, in depths greater than $500 \mathrm{~m}$, and 
Table 1. Statistics relating to the histograms in Figs. 4-6.

\begin{tabular}{cccccc}
\hline Figure & Quantity & mean & st. dev. & $\max$ & $\min$ \\
\hline Figure 4a & $\log \left(D^{+}\right)$ & 0.68 & 0.53 & 2.9 & -1.2 \\
Figure 4b & $\log \left(D^{-}\right)$ & 0.68 & 0.53 & 3.0 & -1.0 \\
Figure 5 & $u_{E u l}$ & 0.028 & 0.24 & 0.81 & -0.69 \\
Figure 5a & $u_{\text {Lag }}$ & 0.033 & 0.28 & 0.75 & -0.60 \\
Figure 5b & $u_{\text {Lag }}$ & 0.043 & 0.21 & 0.69 & -0.47 \\
Figure 5c & $u_{\text {Lag }}$ & 0.24 & 0.21 & 0.70 & -0.59 \\
Figure 6 & $v_{\text {Eul }}$ & 0.012 & 0.22 & 0.73 & -0.88 \\
Figure 6a & $v_{\text {Lag }}$ & 0.0098 & 0.24 & 0.57 & -0.68 \\
Figure 6b & $v_{\text {Lag }}$ & 0.0057 & 0.24 & 0.52 & -0.70 \\
Figure 6c & $v_{\text {Lag }}$ & 0.13 & 0.084 & 0.43 & -0.25 \\
\hline
\end{tabular}

drifters were launched on the same dates $30 \mathrm{~km}$ apart along lines of random orientation centered at that location. In this way, a single experiment consists of launching 58 drifters over the 29 day period. With daily sampling of the drifter data, this results in a maximum of 870 Lagrangian velocity observations in any single experiment. Velocity data from drifters located outside the region of interest were not recorded.

\subsection{Velocity sampling}

The "truth" used for the error metric is the distribution of Eulerian velocities throughout the time period in the region of interest, approximately 180000 measurements. To determine how well the velocities sampled by different drifter launches represent the Eulerian distribution, a quantilequantile comparison of the resulting Lagrangian and Eulerian distributions is made for both meridional and zonal velocity fields. Fifty quantiles are computed from each distribution from which the error metric

$$
\operatorname{Error}_{u}=\frac{1}{50} \Sigma_{i=1}^{50}\left(u_{\text {Lag }}^{i}-u_{E u l}^{i}\right)^{2}
$$

is determined. Here, $u_{L a g}^{i}$ and $u_{E u l}^{i}$ are the $i$ th quantile values for the Lagrangian and Eulerian zonal velocity distributions. A similar error metric Error $v$ is defined for the meridional velocity distributions. Figures 5 and 6 show the PDFs and quantile-quantile plots of the zonal and meridional velocity sampling from the directed launches and the random launches with the lowest and highest errors. The grey background histogram in the sub-figures (a)-(c) is the Eulerian PDF, and the thin solid line is a Gaussian PDF with the same mean and variance for reference. Details of the statistics are given in Table 1 .

For both components of the velocity, the aggregate Eulerian distribution is relatively well represented by a Gaussian with higher skewness seen in the meridional field. Again for either case, the distribution of velocities sampled by the directed launch drifters is remarkably similar to the complete Eulerian distribution indicating that drifter launched in

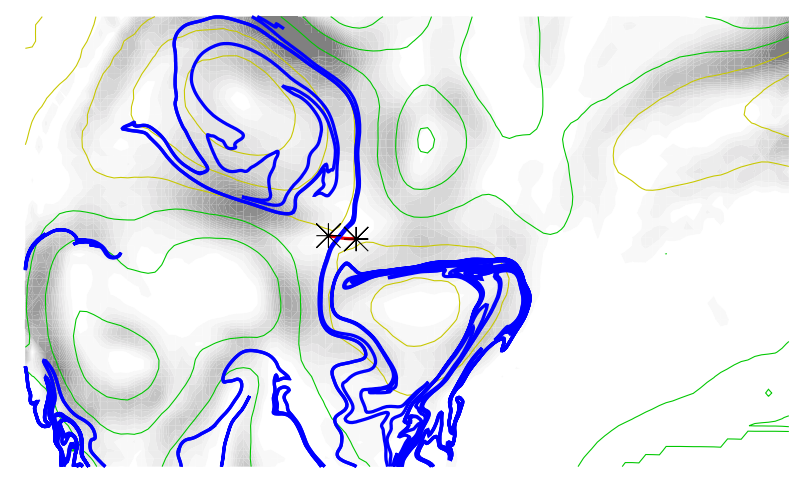

(a) Oct. 19

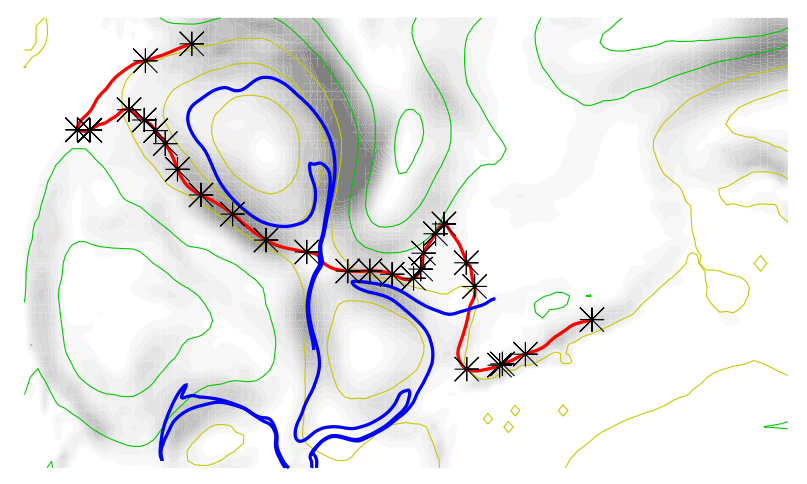

(b) Nov. 2

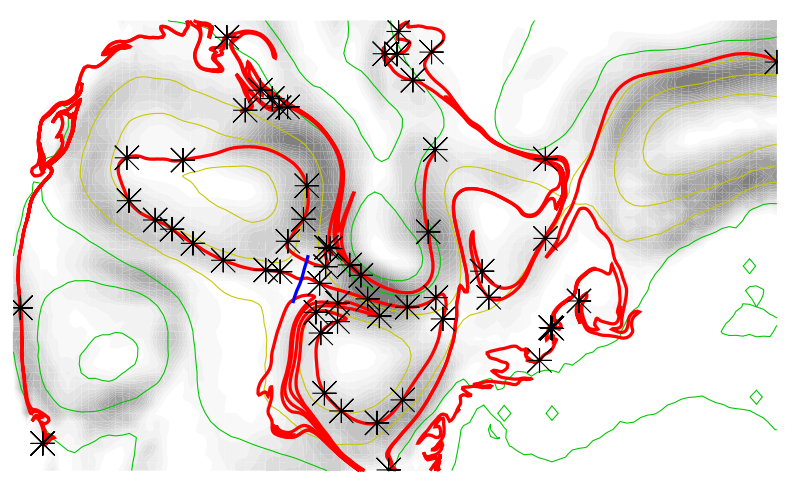

(c) Nov. 16

Fig. 7. Spatial distribution of the drifters $(*)$ for the directed launch experiment shown with respect to the in-flowing (blue) and outflowing (red) material curves emanating from the hyperbolic trajectory of interest. The model height contours (see Fig. 3) overlay the greyscale background which shows the kinetic energy (darkest values exceed $0.5 \mathrm{~m}^{2} / \mathrm{s}^{2}$ ) of the velocity field. 


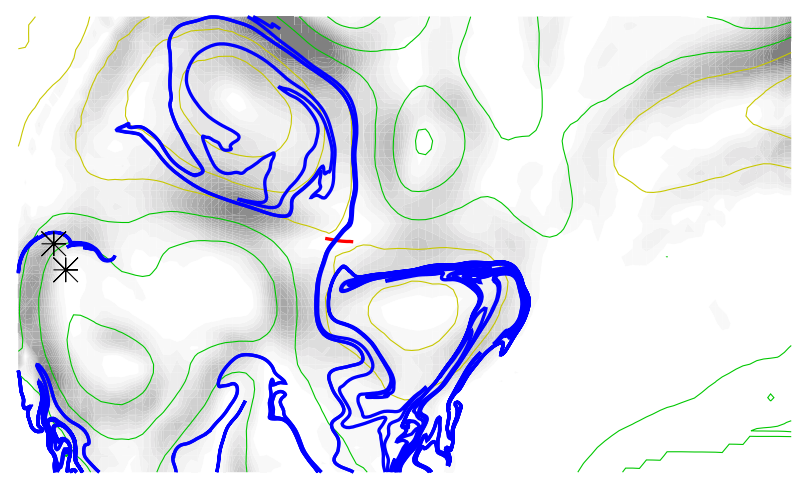

(a) Oct. 19

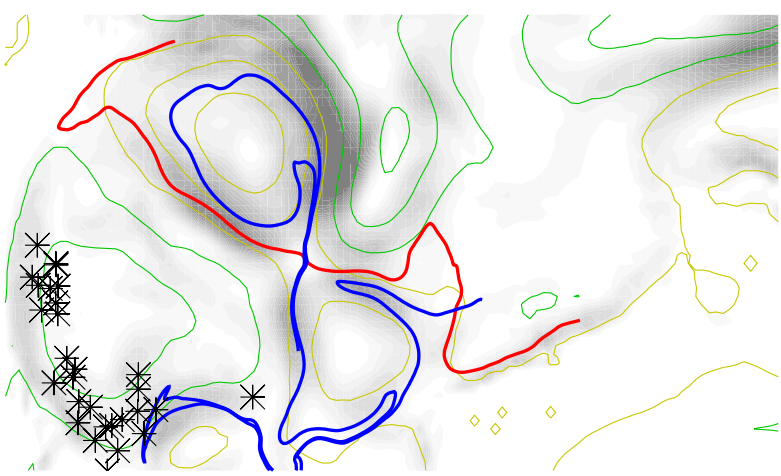

(b) Nov. 2

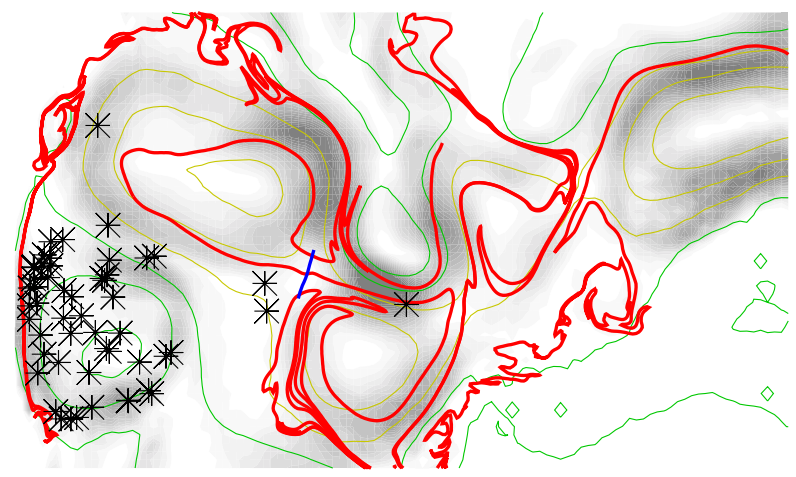

(c) Nov. 16

Fig. 8. Spatial distribution of drifters for the experiment yielding the best zonal velocity sampling. Figure details are the same as for Fig. 7.

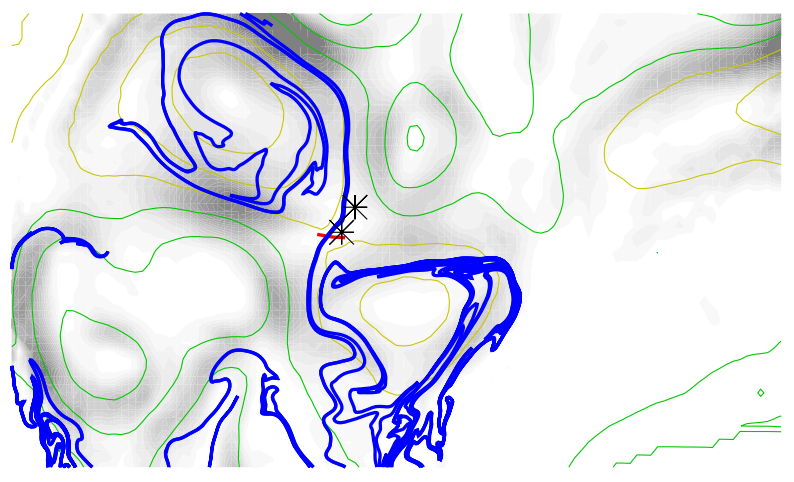

(a) Oct. 19

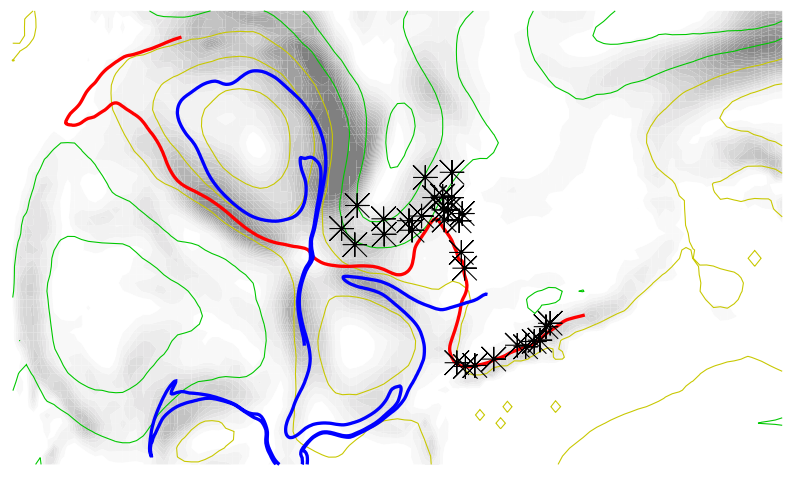

(b) Nov. 2

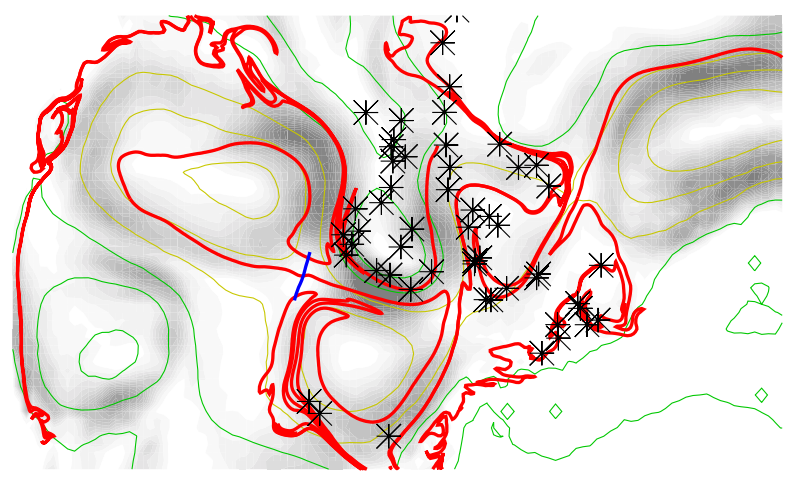

(c) Nov. 16

Fig. 9. Spatial distribution of drifters for the experiment yielding the best meridional velocity sampling. Figure details are the same as for Fig. 7. 


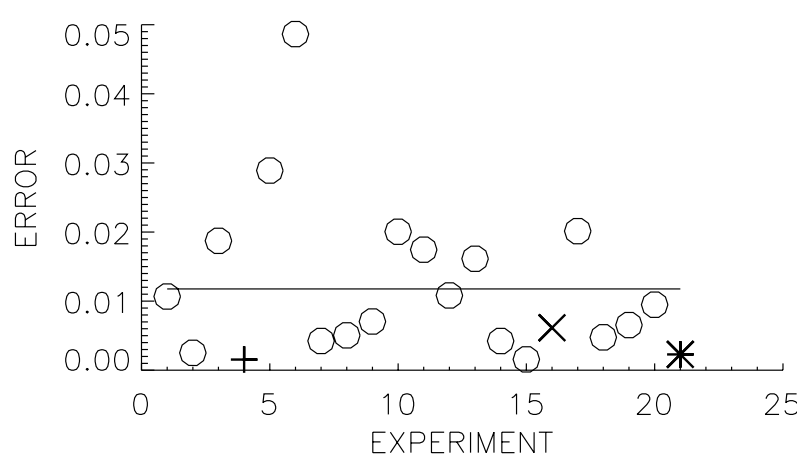

(a) Zonal error

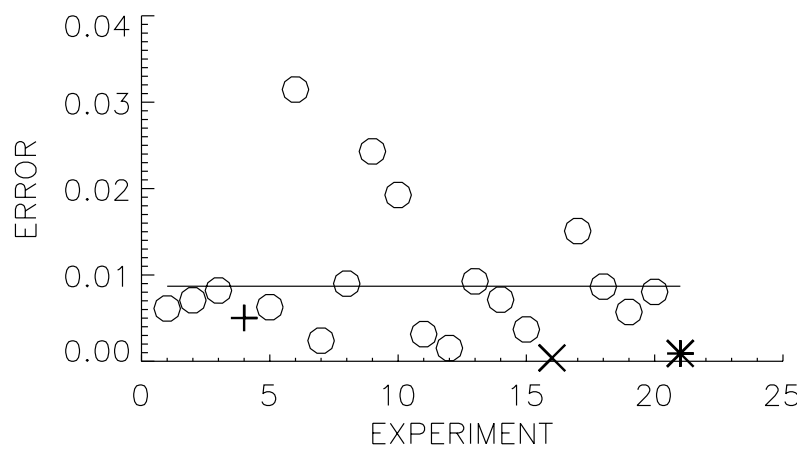

(b) Meridional error

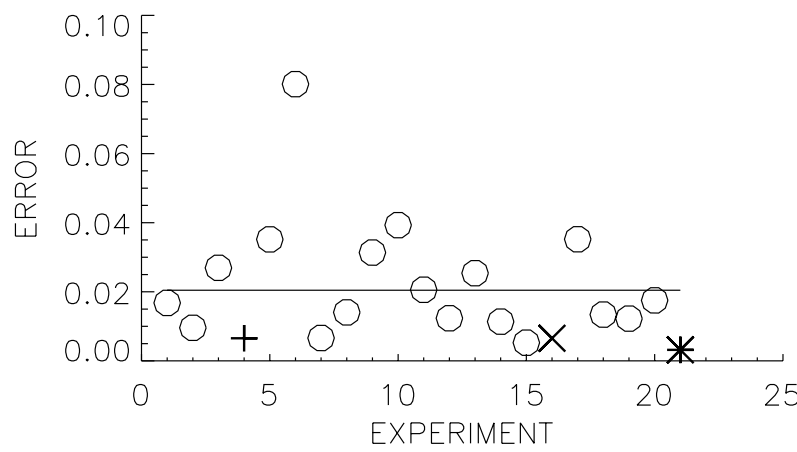

(c) Total error

Fig. 10. The zonal, meridional, and total velocity sampling error for the 20 random launch experiments (1-20) and the directed launch experiment (21, indicated by the asterisk). Experiments with that sampled the zonal and meridional velocities best are indicated by a + and $\times$ respectively. The mean errors are indicated by the solid, horizontal lines. hyperbolic regions preferentially sample the complete Eulerian velocity space.

Launch locations of the random experiments clearly play an important role in the quality of the velocity sampling. Figures 7-9 show the directed and best sampling deployments for the zonal and meridional velocities. Note that the best zonal sampling experiment is different than the best meridional sampling experiment. The background of these plots are contours of the kinetic energy to indicate high and low velocity regions. Also, on these plots are the original manifolds computed from the hyperbolic trajectory used for the directed launch strategy.

The best zonal velocity sampling launch (see Fig. 8) is associated with the cyclone in the southwest corner of the image (see Fig. 1). Indeed, this location placed drifters near the in-flowing manifold of another hyperbolic trajectory that forms as this cyclone splits. The in-flowing manifold of the hyperbolic trajectory shown connects the two saddles, as seen on 19 October (see Fig. 8a). Drifters launched in this experiment are mostly entrained into this cyclone (see Figs. $8 \mathrm{~b}$ and $\mathrm{c}$ ).

The best meridional velocity sampling (see Fig. 9) is achieved with a launch that is remarkably close to the directed launch site. From their initial positions in Fig. 9a, it is clear that western branch of the out-flowing manifold will be well-sampled. However, additional drifters become entrained into a small cyclone to the east of the hyperbolic trajectory (see Figs. 9b and c), which the directed drifters could not enter.

Figure 10 shows the zonal, meridional, and total sampling error of each experiment. Notice the discrepancy between the zonal and meridional sampling accuracies. For example, experiments 3 and 5 sample the zonal velocity significantly worse that the mean sampling error, yet they sample the meridional velocities slightly less than the mean sampling error. Experiment 16 sampled the meridional and experiment 4 the zonal velocities most accurately.

Although the directed launch drifters did not have the least error for either the zonal or meridional velocity samplings, the total error of this launch (0.0032) was significantly better than any of the random launches (the next lowest error was 0.0053 ). Interestingly, the total error of experiments 4 and 16 were nearly identical $(0.0065)$.

\section{Conclusions}

In the western Gulf of Mexico, we have examined how coherent features control both a derived product of the Lagrangian map, two-particle dispersion, and the sampling of the Eulerian velocity by drifters. The dispersion statistics clearly identify the in-flowing and out-flowing manifolds of hyperbolic trajectories associated with the slowly evolving mesoscale eddies in the western Gulf. Drifters launched along the out-flowing direction of a strong hyperbolic trajectory that separates two anti-cyclones in the center of the region were shown to preferentially sample the Eulerian 
velocity distribution over similar, randomly located launch sites. In particular, the directed launch strategy achieved the minimum total sampling error of the combined zonal and meridional velocities.

The conclusion to be drawn from these results is that coherent features such as mesoscale eddies should be taken into account whenever drifter experiments are designed. Hyperbolic regions in the realistic, data assimilating model that contain a hyperbolic trajectory were easily identified. In the ocean, however, specific experiments that not only identify these regions, but calculate drifter separation rates will be an important next step.

Acknowledgements. The authors thank L. Kantha for providing access to the numerical data and A. D. Kirwan for sage advice, insightful comments and gracious hospitality. This work was supported in part by NSF grant ATM-200102. MT was also supported through ONR grant N00014-00-0019. The authors also thank M. Fiadeiro, who inadvertently initiated the velocity sampling research through a brief discussion at the LAPCOD meeting.

Edited by: S. Wiggins

Reviewed by: two referees

\section{References}

Aref, H.: Stirring by chaotic advection, J. Fluid Mech., 192, 115173, 1984.

Blumberg, A. and Mellor, G.: A description of a three-dimensional coastal ocean circulation model, in Three-Dimensional Coastal Ocean Models, edited by N. Heaps, vol. 4, American Geophysical Union, 1987.

Boffetta, G, G., Lacorata, G., Radaelli, and Vulpiani, A.: Detecting barriers to transport: a review of different techniques, Physica D, 159, 58-70, 2001.

Bowman, K.: Manifold geometry and mixing in observed atmospheric flows., preprint, 1999.

Bracco, A., LaCasce, J., and Provenzale, A.: Velocity probability density functions for oceanic floats, J. Phys. Ocean., 30, 461474, 2000

Coulliette, C. and Wiggins, S.: Intergyre transport in a wind-driven, quasigeostrophic double gyre: An application of lobe dynamics, Nonlinear Processses in Geophysics, 7, 59-85, 2000.

Elhmaidi, D., Provenzale, A., and Babiano, A.: Elementary topology of two-dimensional turbulence from a Lagrangian viewpoint and single particle dispersion, J. Fluid Mechanics, 257, 533-558, 1993.

Eremeev, V., Ivanov, L., and Kirwan, A.: Reconstruction of oceanic flow characteristics from quasi-Lagrangian data: 1. Approach and mathematical-methods, J. Geophys. Res-Oceans, 97, 97339742, 1992.

Galperin, B., Kantha, L. H., Hassid, S., and Rosati, A.: A Quasiequilibrium Turbulent Energy Model for Geophysical Flows, J. Atmos. Sci., 45, 55-62, 1988.

Garraffo, Z., Mariano, A., Griffa, A., C., V., and Chassignet, E.: Lagrangian data in a high-resolution numerical simulation of the North Atlantic I. Comparison with in situ drifter data, J. Marine Syst., 29, 157-176, 2001.
Haller, G.: Lagrangian coherent structures and the rate of strain in two-dimensional turbulence., Phys. Fluids, 13, 3365-3385, 2001.

Haller, G. and Poje, A.: Finite time transport in aperiodic flows, Physica D, 119, 352-380, 1998.

Hua, B., McWilliams, J., and Klein, P.: Lagrangian accelerations in geostrophic turbulence, J. Fluid Mech., 366, 87-108, 1998.

Ishikawa, Y. I., Awaji, T., and Akimoto, K.: Successive correction of the mean sea surface height by the simultaneous assimilation of drifting buoy and altimetric data, J. Phys. Oceanog., 26, 23812397, 1996.

Jones, C. and Winkler, S.: Do Invariant Manifolds Hold Water?, in Handbook of Dynamical Systems III: Towards Applications, edited by G. B.Fiedler and N.Kopell, Elsevier, 2001.

Kamachi, M. and O'Brien, J.: Continuous assimilation of drifting buoy trajectory into an equatorial Pacific ocean model, J. Mar. Sys., 6, 159-178, 1995.

Kantha, L. H. and Clayson, C. A.: An Improved Mixed Layer Model for Geophysical Applications, J. Geo. Res., 99, 25235 25 266, 1994.

Kantha, L. H., K.Choi, J., Leben, R., Cooper, C., Vogel, M., and Feeney, J.: Hindcasts and Real-time Nowcast/Forecasts of Currents in the Gulf of Mexico, in Offshore Technology Conference, Houstan, Texas, 1999.

Kuznetsov, L., Toner, M., Kirwan, A. D., Jones, C., Kantha, L., and Choi, J.: The Loop Current and adjacent rings delineated by Lagrangian analysis., J. Mar. Res., 60, 2002.

Lapeyre, G., Klien, P., and Hua, B.: Does the tracer gradient vector align with the strain eigenvectors in 2D turbulence?, Phys. Fluids, 11, 3729-3737, 1999.

Lebovitz, N. R. and Neishtadt, A.: Slow evolution in perturbed Hamiltonian systems, Stud. Appl. Math., 92, 127-144, 1994.

Lewis, J. and Kirwan, A.: Some observations of ring-topography and ring-ring interatcions in the Gulf of Mexico, J. Geo. Res.Oceans, 90, 9017-9028, 1985.

Lopez, J. W. and Kantha, L. H.: A data-assimilative numerical model of the North Indian Ocean, J. Atmos. Ocean. Tech., 17, 1525-1540, 2000.

MacKay, R., Meiss, J., and Percival, I.: Transport in Hamiltonian Systems., Phys. D, 13, 55-81, 1984.

Miller, P., Jones, C., Rogerson, A., and Pratt, L.: Quantifying transport in numerically generated velocity fields, Physica D, 110, 105-122, 1997.

Miller, P., Pratt, L., Helfrich, K., and Jones, C.: Chaotic transport of mass and potential vorticity for an island recirculation, J. Phys. Oceanogr., 32, 80-102, 2002.

Molcard, A., Piterbarg, L., Griffa, A., Ozgokmen, T. M., and Mariano, A. J.: Assimilation of drifter positions for the reconstruction of the Eulerian circulation field, J. Geophys. Res., in Press, 2003.

Okubo, A.: Horizontal dispersion of floatable particles in the vicinity of volcity singularities such as convergences, Deep Sea Res. 17, 445-454, 1970.

Pierrehumbert, R.: Chaotic mixing of tracer and vorticity by modulated traveling Rossby waves, Geophys. Astr. Fluid Dynamics, 58, 285-320, 1991.

Poje, A. and Haller, G.: Geometry of cross-stream mixing in a double-gyre ocean model, J. Phys. Oceanogr., 29, 1649-1665, 1999.

Poje, A., Haller, G., and Mezic̀, I.: The geometry and statistics of mixing in aperiodic flows, Phys. Fluids, 11, 2963-2968, 1999.

Poje, A. C., Toner, M., A. D. Kirwan, J., and Jones, C. K. R. T.: Drifter Launch Strategies Based on Lagrangian Templates, J. 
Phys. Oceanogr., 32, 1855-1869, 2002.

Provenzale, A.: Transport by coherent barotropic vortices, Annual Rev. Fluid Mech., 31, 55-93, 1999.

Ridderinkkhof, H. and Loder, J.: Lagrangian characterization of circulation over submarine banks with application to the Gulf of Maine, J. Phys. Oceanogr., 24, 1184-1200, 1994.

Rom-Kedar, V., Leonard, A., and Wiggins, S.: An Analytical Study of Transport, Mixing and Chaos in an Unsteady Vortical Flow, J. Fluid Mech., 214, 347-394, 1990.

Rupolo, V., Hua, B., Provenzale, A., and Artale, V.: Lagrangian velocity spectra at $700 \mathrm{~m}$ in the western North Atlantic, J. Phys Oceanogr., 26, 1591-1607, 1996.

Toner, M., Kirwan, A., Kantha, L., and Choi, J.: Can general circulation models be assessed and enhanced with drifter data?, J. Geo. Res.-Oceans, 106, 1366-1383, 2001a.
Toner, M., Poje, A., Kirwan, A., Jones, C., Liphardt, B., and Grosch, C.: Reconstructing Basin-Scale Eulerian Velocity Fields from Simulated Drifter Data, J. Phys. Oceanogr., 31, 1361-1376, 2001b.

Toner, M., Kirwan, A., Poje, A., Kantha, L., Müller-Karger, F. E., and Jones, C.: Chlorophyll dispersal by eddy-eddy interactions in the Gulf of Mexico, J. Geophys. Res., 108, 3105, doi:10.1029/2002JC001499, 2003.

Weiss, J.: The dynamics of enstrophy transfer in 2-dimensional hydrodynamics, Physica D, 48, 273-294, 1991. 